\title{
Correction to: Design of a thermal desalination system with spray flash drum and condenser under vacuum based on droplet dynamics
}

\author{
Arnab Karmakar ${ }^{1}$ (I) $\cdot{\text { Dipanjan } K^{1}{ }^{1} \cdot \text { Adil Ahmad }^{1} \cdot \text { Sumit Kumar Jana }}^{1}$
}

Published online: 27 November 2021

c) Springer-Verlag GmbH Germany, part of Springer Nature 2021

\section{Correction to: Heat and Mass Transfer https://doi.org/10.1007/s00231-021-03152-4}

Our online manuscripts has some typographical errors in editing, mainly dash (-) symbols are missing. The corrections are provided in a table with their errors and locations. The line numbers of the erratum are based on the Author's proof.

\begin{tabular}{llll}
\hline $\begin{array}{l}\text { Location of error with } \\
\text { section and line numbers } \\
\text { in Author's proof }\end{array}$ & $\begin{array}{l}\text { Error-texts } \\
\text { (erratum) to be } \\
\text { corrected }\end{array}$ & Corrected texts \\
\hline Section:1 & Line: 163 & of $0.1100 \mu \mathrm{m}$ & $0.1-100 \mu \mathrm{m}$ \\
& Line: 184 & $0.1100 \mu \mathrm{m}$ & $0.1-100 \mu \mathrm{m}$ \\
& Line: 217 & $4060{ }^{\circ} \mathrm{C}$ & $40-60{ }^{\circ} \mathrm{C}$ \\
& Line: 218 & $250{ }^{\circ} \mathrm{C}$ & $2-50{ }^{\circ} \mathrm{C}$ \\
Section:2 & Line: 219 & $100,200 \mu \mathrm{m}$ & $100-200 \mu \mathrm{m}$ \\
& Line: 237 & $40{ }^{\circ} \mathrm{C} 60{ }^{\circ} \mathrm{C}$ & $40{ }^{\circ} \mathrm{C}-60{ }^{\circ} \mathrm{C}$ \\
Section:3 & Line: 245 & $1,002,000 \mu \mathrm{m}$ & $100-2000 \mu \mathrm{m}$ \\
& Line: 260 & $00.04 \mathrm{~kg} / \mathrm{kg}$ & $0-0.04 \mathrm{~kg} / \mathrm{kg}$ \\
& Line: 318 & $0120 \mathrm{gm} / \mathrm{kg}$ & $0-120 \mathrm{gm} / \mathrm{kg}$ \\
& Line:327 & $T_{\text {sat }}=T_{\mathrm{F}} \Delta T_{\text {sat }}$ & $T_{\text {sat }}=T_{\mathrm{F}}-\Delta T_{\text {sat }}$ \\
& Line:337 & $250{ }^{\circ} \mathrm{C}$ & $2-50{ }^{\circ} \mathrm{C}$ \\
& Line 338 & $00.040 \mathrm{~kg} / \mathrm{kg}$ & $0-0.040 \mathrm{~kg} / \mathrm{kg}$ \\
& Line 338 & $4060{ }^{\circ} \mathrm{C}$ & $40-60{ }^{\circ} \mathrm{C}$ \\
& Line:343 & $0.358 .37 \%$ & $0.35-8.37 \%$ \\
& Line:348 & $3050{ }^{\circ} \mathrm{C}$ & $30-50{ }^{\circ} \mathrm{C}$ \\
Line:354 & $2632{ }^{\circ} \mathrm{C}$ & $26-32{ }^{\circ} \mathrm{C}$ \\
Line:358 & $2030 \%$ & $20-30 \%$ \\
\hline
\end{tabular}

The original article can be found online at https://doi.org/10.1007/ s00231-021-03152-4.

Arnab Karmakar

arnabkarmakar@bitmesra.ac.in

1 Department of Chemical Engineering, Birla Institute

of Technology, Mesra, Jharkhand 835215, India

\begin{tabular}{|c|c|c|c|}
\hline \multicolumn{2}{|c|}{$\begin{array}{l}\text { Location of error with } \\
\text { section and line numbers } \\
\text { in Author's proof }\end{array}$} & $\begin{array}{l}\text { Error-texts } \\
\text { (erratum) to be } \\
\text { corrected }\end{array}$ & Corrected texts \\
\hline \multicolumn{2}{|c|}{ Heading of Table 1} & $\begin{array}{l}\text { Yield calculation } \\
\text { based } \\
\text { on material and } \\
\text { energy balance } \\
\text { equations of the } \\
\text { flash drum at } \\
\text { saline feed water } \\
\text { temperature } \\
T_{F}=4060^{\circ} \mathrm{C} \text {, } \\
\text { salinity s }=0.035 \\
\& 0.04 \mathrm{~kg} / \mathrm{kg}, \\
\text { feed flow rate } \\
F=40,000 \mathrm{~kg} / \mathrm{day} \\
\text { and at different } \\
\text { supersaturation } \\
\left(250{ }^{\circ} \mathrm{C}\right)\end{array}$ & $\begin{array}{l}\text { Yield calculation } \\
\text { based } \\
\text { on material and } \\
\text { energy balance } \\
\text { equations of the } \\
\text { flash drum at } \\
\text { saline feed water } \\
\text { temperature } T_{F} \\
=40-60{ }^{\circ} \mathrm{C} \text {, } \\
\text { salinity } \mathrm{s}=0.035 \\
\& 0.04 \mathrm{~kg} / \mathrm{kg}, \\
\text { feed flow rate } \\
F=40,000 \mathrm{~kg} / \mathrm{day} \\
\text { and at different } \\
\text { supersaturation } \\
\left(2-50{ }^{\circ} \mathrm{C}\right)\end{array}$ \\
\hline Section: 4.1 & Line: 414 & ISO 0206 & ISO $02-06$ \\
\hline \multirow[t]{10}{*}{ Section: 4.2} & $\begin{array}{r}\text { Line: } \\
530\end{array}$ & $\begin{array}{l}\text { is the surface } \\
\text { temperature }\end{array}$ & $\begin{array}{c}T_{s} \text { is the surface } \\
\text { temperature }\end{array}$ \\
\hline & Line: 533 & $10^{3} 10^{5}{ }^{\circ} \mathrm{C}$ & $10^{-3}-10^{-5}{ }^{\circ} \mathrm{C}$ \\
\hline & Line: 535 & $220^{\circ} \mathrm{C}$ & $2-20^{\circ} \mathrm{C}$ \\
\hline & Line: 535 & $250,500 \mu \mathrm{m}$ & $250-500 \mu \mathrm{m}$ \\
\hline & Line: 613 & $5060^{\circ} \mathrm{C}$ & $50-60{ }^{\circ} \mathrm{C}$ \\
\hline & Line: 614 & $2050^{\circ} \mathrm{C}$ & $20-50^{\circ} \mathrm{C}$ \\
\hline & Line: 614 & $\mathrm{c} 5 \mathrm{c} 8$ & $c 5-c 8$ \\
\hline & Line: 621 & $5060^{\circ} \mathrm{C}$ & $50-60^{\circ} \mathrm{C}$ \\
\hline & Line: 621 & $2050^{\circ} \mathrm{C}$ & $20-50^{\circ} \mathrm{C}$ \\
\hline & Line: 622 & $\mathrm{c} 5 \mathrm{c} 8$ & $c 5-c 8$ \\
\hline \multicolumn{2}{|c|}{ Heading of Fig. 6} & $\begin{array}{l}\text { Surface temperature } \\
\text { dynamics of a } \\
\text { saline water } \\
\text { droplet at higher } \\
\text { feed temperature } \\
\left(5060^{\circ} \mathrm{C}\right) \text { and } \\
\text { higher } \\
\text { supersaturation } \\
\left(2050^{\circ} \mathrm{C}\right)\end{array}$ & $\begin{array}{l}\text { Surface temperature } \\
\text { dynamics of a } \\
\text { saline water } \\
\text { droplet at higher } \\
\text { feed temperature } \\
\left(50-60^{\circ} \mathrm{C}\right) \\
\text { and higher } \\
\text { supersaturation } \\
\left(20-50^{\circ} \mathrm{C}\right)\end{array}$ \\
\hline
\end{tabular}




\begin{tabular}{|c|c|c|c|c|c|c|c|}
\hline \multicolumn{2}{|c|}{$\begin{array}{l}\text { Location of error with } \\
\text { section and line numbers } \\
\text { in Author's proof }\end{array}$} & $\begin{array}{l}\text { Error-texts } \\
\text { (erratum) to be } \\
\text { corrected }\end{array}$ & Corrected texts & \multicolumn{2}{|c|}{$\begin{array}{l}\text { Location of error with } \\
\text { section and line numbers } \\
\text { in Author's proof }\end{array}$} & $\begin{array}{l}\text { Error-texts } \\
\text { (erratum) to be } \\
\text { corrected }\end{array}$ & Corrected texts \\
\hline \multicolumn{2}{|c|}{ Heading of Fig. 7} & $\begin{array}{l}\text { The change of } \\
\text { droplet radius } \\
\text { with time at } \\
\text { higher feed } \\
\text { temperature } \\
\left(5060^{\circ} \mathrm{C}\right) \text { and } \\
\text { higher } \\
\text { supersaturation } \\
\left(2050{ }^{\circ} \mathrm{C}\right)\end{array}$ & $\begin{array}{l}\text { The change of } \\
\text { droplet radius } \\
\text { with time at } \\
\text { higher feed } \\
\text { temperature } \\
\left(50-60^{\circ} \mathrm{C}\right) \\
\text { and higher } \\
\text { supersaturation } \\
\left(20-50^{\circ} \mathrm{C}\right)\end{array}$ & \multirow{2}{*}{\multicolumn{2}{|c|}{ Heading of Table 5}} & $\begin{array}{l}\text { Estimated value } \\
\text { of overall heat } \\
\text { transfer coefficient } \\
U, \text { shell side } \\
\text { condensation film } \\
\text { coefficient } h_{c} \text {, } \\
\text { and tube side heat } \\
\text { transfer coefficient } \\
\text { for cooling water }\end{array}$ & $\begin{array}{l}\text { Estimated value of } \\
\text { overall heat transfer } \\
\text { coefficient } U \text {, shell } \\
\text { side condensation } \\
\text { film coefficient } h_{c} \text {, } \\
\text { and tube side heat } \\
\text { transfer coefficient } \\
\text { for cooling water } h_{w} \\
\text { at feed temperature }\end{array}$ \\
\hline \multirow[t]{4}{*}{ Section: 4.2} & $\begin{array}{r}\text { Line: } \\
628\end{array}$ & $0.51 \mathrm{~s}$ & $0.5-1 \mathrm{~s}$ & & & $\begin{array}{l}h_{w} \text { at feed } \\
\text { temperature } \\
4060{ }^{\circ} \mathrm{C}\end{array}$ & $40-60^{\circ} \mathrm{C}$ \\
\hline & $\begin{array}{r}\text { Line: } \\
652\end{array}$ & $9.2^{\circ} \mathrm{C}$ & $-9.2^{\circ} \mathrm{C}$ & \multirow[t]{3}{*}{ Section:6 } & Line: & $69.2484 .59 \%$ & $69.24-84.59 \%$ \\
\hline & Line: & $17^{\circ} \mathrm{C}$ & $-17^{\circ} \mathrm{C}$ & & $\begin{array}{l}808 \\
\text { Line: }\end{array}$ & $100.150 \mu \mathrm{m}$ & $100-150 \mu \mathrm{m}$ \\
\hline & Line: & $20^{\circ} \mathrm{C}$ & $-20^{\circ} \mathrm{C}$ & & 815 & & \\
\hline
\end{tabular}

The original article has been corrected.

$\begin{array}{rrll}\text { Section:5 } & \text { Line: } & 0.00170 .017 \mathrm{~kg} / \mathrm{s} & 0.0017-0.017 \mathrm{~kg} / \mathrm{s} \\ 695 & & \\ & \text { Line: } & 2025^{\circ} \mathrm{C} & 20-25^{\circ} \mathrm{C} \\ 699 & & \end{array}$

Publisher's Note Springer Nature remains neutral with regard to jurisdictional claims in published maps and institutional affiliations. 